\title{
Progress in the design of the superconducting magnets for the EU DEMO
}

\author{
V. Corato ${ }^{\mathrm{a}, *}$, T. Bagni ${ }^{\mathrm{b}}$, M.E. Biancolini ${ }^{\mathrm{c}}$, R. Bonifetto ${ }^{\mathrm{d}}$, P. Bruzzone $^{\mathrm{e}}$, N. Bykovsky $^{\mathrm{e}}$, D. Ciazynski $^{\mathrm{f}}$, \\ M. Coleman ${ }^{g}$, A. della Corte ${ }^{a}$, A. Dembkowska ${ }^{h}$, A. Di Zenobio ${ }^{a}$, M. Eisterer ${ }^{i}$, W.H. Fietz ${ }^{j}$, \\ D.X. Fischer ${ }^{1}$, E. Gaio $^{k}$, L. Giannini ${ }^{\mathrm{a}}$, F. Giorgetti ${ }^{\mathrm{c}}$, R. Heller ${ }^{\mathrm{j}}$, I. Ivashov ${ }^{1}$, B. Lacroix ${ }^{\mathrm{f}}$,

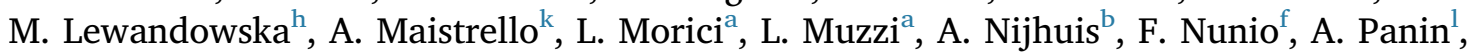 \\ X. Sarasola ${ }^{\mathrm{e}}$, L. Savoldi ${ }^{\mathrm{d}}$, K. Sedlak ${ }^{\mathrm{e}}$, B. Stepanov ${ }^{\mathrm{e}}$, G. Tomassetti ${ }^{\mathrm{a}}$, A. Torre ${ }^{\mathrm{f}}$, S. Turtù $^{\mathrm{a}}$, \\ D. Uglietti ${ }^{e}$, R. Vallcorba ${ }^{f}$, K.-P. Weiss ${ }^{j}$, R. Wesche ${ }^{\mathrm{e}}$, M.J. Wolf ${ }^{\mathrm{j}}$, K. Yagotintsev $^{\mathrm{b}}$, L. Zani ${ }^{\mathrm{f}}$, \\ R. Zanino ${ }^{\mathrm{d}}$
}

a ENEA, 00044, Frascati, Italy

${ }^{\mathrm{b}}$ Univ. of Twente, 7522, Enschede, Netherlands

${ }^{\mathrm{c}}$ University of Rome Tor Vergata, 00133, Rome, Italy

d NEMO group, Dipartimento Energia, Politecnico di Torino, 10129, Torino, Italy

${ }^{\mathrm{e}} \mathrm{EPFL} / \mathrm{SPC}$, 5232, Villigen, Switzerland

${ }^{\mathrm{f}}$ Commissariat à l'Energie Atomique et aux Energies Alternatives, France

${ }^{\mathrm{g}}$ EUROfusion, 85748, Garching bei München, Germany

${ }^{\mathrm{h}}$ West Pomeranian University of Technology, 70310, Szczecin, Poland

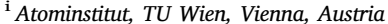

${ }^{j}$ KIT, 76344, Eggenstein-Leopoldshafen, Germany

${ }^{\mathrm{k}}$ Consorzio RFX, 35127, Padova, Italy

${ }^{1}$ IEK-4 - Plasma Physics, Forschungszentrum Juelich GmbH, D52425, Juelich, Germany

\section{A R T I C L E I N F O}

\section{Keywords:}

Nuclear fusion

DEMO

Superconducting magnets

CICC

\begin{abstract}
A B S T R A C T
In the framework of the DEMOnstration fusion power plant (DEMO) design coordinated by the EUROfusion consortium, a pre-conceptual design of the superconducting magnet system has been developed. For the toroidal field coils (TFCs), three winding pack (WP) options have been proposed; exploring different winding approaches (pancakes vs. layers), and manufacturing techniques (react \& wind vs. wind \& react $\mathrm{Nb}_{3} \mathrm{Sn}$ ). Thermal-hydraulic and mechanical analyses on the three WPs have produced encouraging results, with some critical issues to be solved in future studies and optimizations. The experimental tests on TF prototype short sample conductors have demonstrated a limited performance degradation with electro-magnetic cycles and significantly lower effective strains than most of the large-size $\mathrm{Nb}_{3} \mathrm{Sn}$ conductors reported in literature. The toroidal field quench protection circuit has been studied, starting from different topologies and focusing on the most promising one. Two designs are also presented for the central solenoid magnet, with preliminary evaluations on the AC losses during the plasma breakdown. Finally, the design of a TF winding pack based on HTS conductors and the experimental tests on "fusion-relevant" HTS cables are illustrated.
\end{abstract}

\section{Introduction}

In the framework of the Roadmap to Fusion Electricity Horizon 2020 [1], EUROfusion consortium is coordinating a comprehensive design study of a DEMOnstration fusion power plant (DEMO) [2] with the aim to demonstrate that a fusion reactor can produce net electrical power and supply energy to the grid.

This paper reports on the latest status of the pre-conceptual design of the DEMO magnet system, focusing on the progress since the results already mentioned in [3]. In Section 2 three configurations for the toroidal field (TF) winding packs are proposed, based on different winding schemes (layer vs. pancake) and $\mathrm{Nb}_{3} \mathrm{Sn}$ manufacturing technology (wind \& react (W\&R) vs. react \& wind (R\&W)). For each configuration thermal-hydraulic and mechanical analyses are presented, as well as the results of the R\&D activity on short-size samples, where available. Preliminary studies on different possible topologies of the TF

\footnotetext{
* Corresponding author.

E-mail address: valentina.corato@enea.it (V. Corato).
} 
quench protection circuit (QPC) are presented in Section 3. Section 4 reports on two proposals for the design and preliminary analyses of the central solenoid (CS) coils. The design and the experimental activity on High Temperature Superconductor (HTS) conductors relevant to fusion program are illustrated in Section 5.

\section{TF coils pre-conceptual design and R\&D}

In this section three designs for the toroidal field coils are proposed: WP\#1, WP\#2, and WP\#3. All designs refer to the 2015 reference baseline for the DEMO reactor [4]. Some of the short length samples, already manufactured and tested, are based on the previous design (2013 reference baseline [5]), characterized by different requirements. The scope of these R\&D programs and experimental campaigns was to demonstrate the feasibility and performances of new technologies, rather than qualify a conductor layout for a specific set of requirements.

\subsection{TF WP\#1 design}

WP\# $1[6,7]$ is based on the R\&W method for $\mathrm{Nb}_{3} \mathrm{Sn}$ magnets, in which the conductor is wound after the heat treatment, which is carried out without the stainless steel (SS) conduit and electrical insulation. The WP is wound in single layers (SL); this approach allows the grading of both superconductor (SC) and SS cross-sections in the different layers, depending on the magnetic field and the local stress value, respectively. In particular, with decreasing distance from the plasma the SC content decreases, thus optimizing the use of SC as function of the local magnetic field, whereas the jacket thickness increases to withstand the higher mechanical stresses $[8,9]$.

As shown in Fig. 1(a), the WP layout consists of 12 layers by 19 turns (17 turns in the last layer), i.e. 226 turns in total, allowing a conductor operating current of $63.3 \mathrm{kA}$.

The conductor, named RW2, has a flat shape, to keep the SC strands close to the neutral bending axis, and two SS shells, assembled by longitudinal laser welding. Two solid profiles of $\mathrm{Cu} / \mathrm{CuNi}$ mixed matrix are used as stabilizer. The layout of the RW2 conductor is listed in Table 1, in comparison with the main parameters of the first prototype, namely RW1, designed for the previous DEMO reference baseline [5].

The thermo-hydraulic analyses $[10,11]$ have been carried out on the proposed WP design considering a nuclear heating (NH) load $\mathrm{P}_{\mathrm{NH}}(\mathrm{R})=50 \exp \left(-\frac{\mathrm{r}}{0.14}\right) \mathrm{W} / \mathrm{m}^{3}$, as a function of the radial distance $\mathrm{r}$
Table 1

Main parameters the RW1 and RW2 conductors for the highest field layer.

\begin{tabular}{lll}
\hline Layout & RW1 & RW2 \\
\hline Operating current [kA] & 82.4 & 63.3 \\
Peak operating field [T] & 13.3 & 12.23 \\
Width x height [mm x mm] & $100 \times 34$ & $61.5 \times 32.1$ \\
Strand diameter [mm] & 1.5 & 1.2 \\
Number SC strands & 306 & 234 \\
Number Cu strands & 17 & 13 \\
SC cable size [mm x mm] & $62.0 \times 12.3$ & $35 \times 11$ \\
Void fraction in cable [\%] & 27 & 23 \\
Jcopper [A/mm²] & 119 & 90 \\
Jnon-copper [A/mm ${ }^{2}$ ] & 305 & 478 \\
Central strip in flat cable & none & steel \\
Steel cross-section [mm ${ }^{2}$ ] & 2060 & 893.5 \\
Conduit inner radius [mm] & 3 & 8 \\
\hline
\end{tabular}

(in $\mathrm{m}$ ) from the plasma facing wall [12].

The conductor is modelled as a 1-D system of several parallel components - three thermal components (strands, $\mathrm{Cu} / \mathrm{CuNi}$ stabilizer, and steel jacket) and three hydraulic components ( $\mathrm{He}$ in the bundle, $\mathrm{He}$ in the triangular side cooling channels, and $\mathrm{He}$ in the upper rectangular channel). Heat transfer between the solid components is set to $500 \mathrm{~W} /$ $\left(\mathrm{m}^{2} \mathrm{~K}\right)$, where the contact surface between strands and copper stabilizer is assumed to be $1 / 5$ of the overall boundary area. The heat transfer coefficient between helium and solid components in turbulent flow is derived from the Dittus-Boelter correlation [13]. The friction correlations and heat transfer coefficients in laminar regime, which may become relevant in part of the conductor during quench transient, are also specified in [13]. In normal conditions, the temperature margin $\Delta T_{m \mathrm{arg}}^{\mathrm{min}}$ of all conductors exceeds the required $1.5 \mathrm{~K}$ minimum value [14]. In off-normal conditions, the quench hot spot temperature, $\mathrm{T}_{\mathrm{hs}}$, is safely below the acceptable limit of $150 \mathrm{~K}$, that guarantees acceptable thermal stress in the winding pack, due to the thermal gradients. Below $150 \mathrm{~K}$, materials have a low thermal expansion and $150 \mathrm{~K}$ is therefore selected as the maximum temperature that may be reached by the conductor jacket.

Concerning mechanical analysis, critical locations are mapped on the curved leg (outer leg) and the straight one (inboard leg), indicated as points 1 and 2, respectively, in Fig. 2(a). The stress acceptance by stress linearization is stated considering several paths that cross the

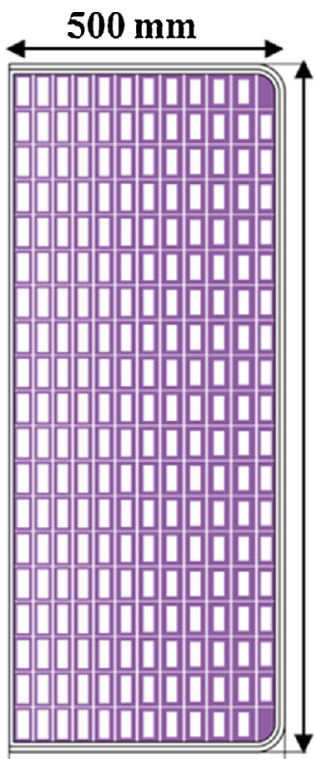

(a) (b)
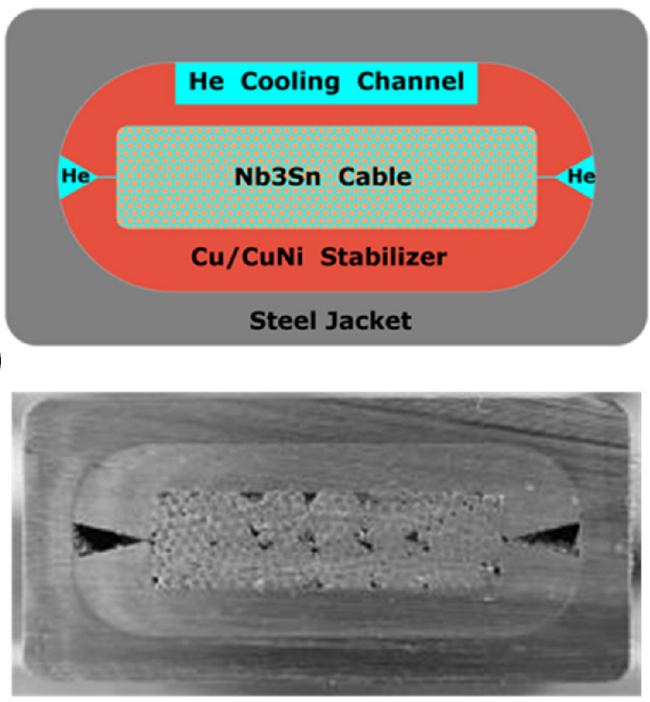

(c)

Fig. 1. (a) TF WP\#1 configuration (b) Cross-section of the RW1 conductor (c) Cross-section of the RW2 conductor. 


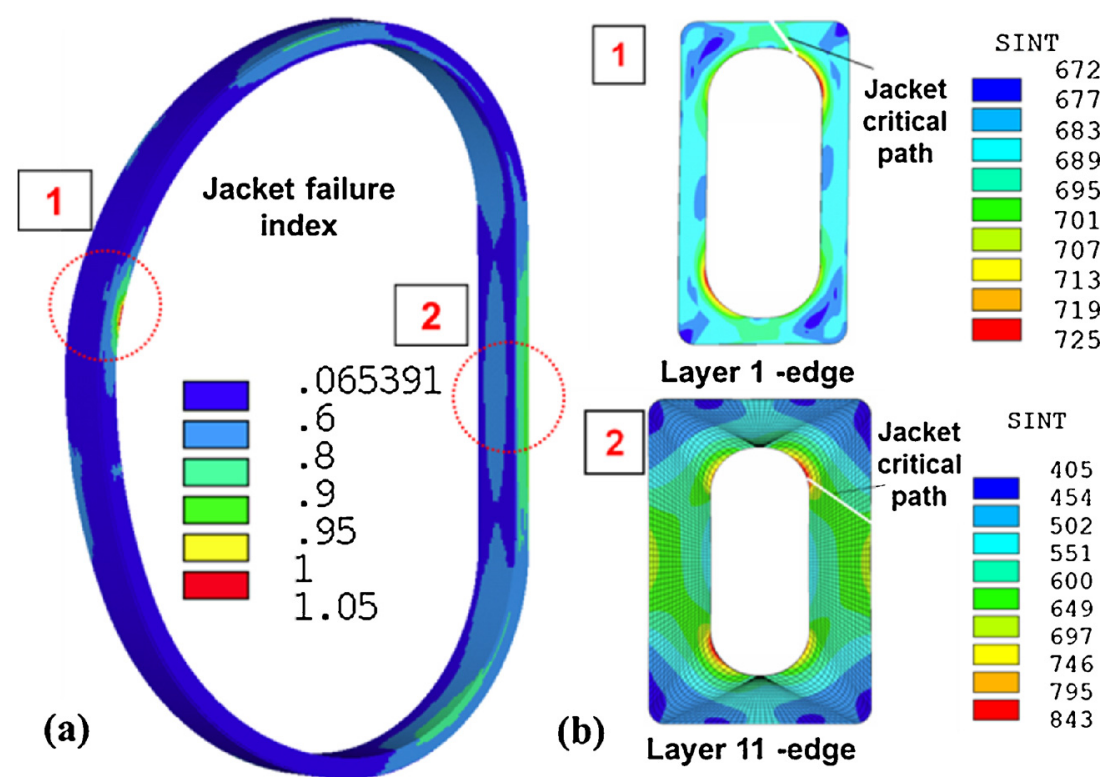

Fig. 2. (a) Structurally critical locations in TF WP\#1 (b) Conductor cross sections and jacket critical paths.

thickness of the jacket. The critical path is the one which present the less margin in regards of the allowable stress. Jacket critical paths are shown in Fig. 2b, and results are here summarized. On the outboard, mostly due to the coil out-of-plane bending, the primary membrane stress for WP\#1 (692 MPa) slightly exceeds the allowable static stress for chosen structural steel ( $667 \mathrm{MPa}$, that is $2 / 3$ of the yield strength of the material). On the inboard leg, where radial and toroidal compressions act in addition to poloidal tension, there are no issues with the jacket static strength. In both legs the sum of the membrane stress plus bending calculated over the critical path is below the static limit of $867 \mathrm{MPa}$, that is $1.3 \times 2 / 3$ of the yield strength of the material. To study the critical locations in insulation, the shear stress coupled with compression has been considered. The insulation static strength is not that critical, but the cyclic shear stress could be an issue for the WP\#1 coil, since the layout does not have enough bending stiffness in response to in-plane and out-of-plane loading.

As a possible countermeasure, the coil in-plane bending may be decreased by re-shaping it, whereas the coil lateral bending is decreased by either stiffening the outer inter-coil structures (OIS) or by increasing the coil case bending stiffness.

Two short-length samples, namely RW1 and RW2 as reported above, were manufactured and tested in the SULTAN test facility [15]. The DC test includes the measurement of the critical current, $I_{c}$, and of the current sharing temperature, $\mathrm{T}_{\mathrm{cs}}$, before and after 1100 load cycles and a warm-up/cool-down (WUCD).

Results after the WUCD of the 2016 experimental campaign for RW1 sample are reported in Fig. 3 [6]. The "effective strain", $\varepsilon_{\text {eff, }}$ is the parameter to be used in the strand scaling law to fit the conductor performance. For RW1, the black and red dashed lines in Fig. 3 give the performance assessment at $\varepsilon=-0.28 \%$ and $\varepsilon=-0.35 \%$ for the left and right legs of the SULTAN sample, respectively. For a given current and field, the temperature at which the average electric field over the cable cross section reaches $0.1 \mu \mathrm{V} / \mathrm{cm}$ is defined as $\mathrm{T}_{\mathrm{cs}}$. At the nominal operation point $\left(\mathrm{I}_{\mathrm{op}}=82.4 \mathrm{kA}, \mathrm{B}_{\mathrm{eff}}=13.3 \mathrm{~T}\right) \mathrm{T}_{\mathrm{cs}}$ is between 7.1-7.3 K, that guarantees a $\Delta T_{m \mathrm{arg}}^{\min }$ higher than the required $1.5 \mathrm{~K}$ minimum value.

Concerning the RW2 sample [7], the nominal operating point $\left(\mathrm{I}_{\mathrm{op}}=63.3 \mathrm{kA}, \mathrm{B}_{\text {eff }}=12.23 \mathrm{~T}\right)$ cannot be achieved in the SULTAN test facility. The cyclic loading is thus applied at the equivalent transverse load condition of $70 \mathrm{kA}$ and $10.9 \mathrm{~T}$. The $\mathrm{T}_{\mathrm{cs}}$ at the nominal operating point is extrapolated, providing a value of about $6.4 \mathrm{~K}$. The evolution of the $\mathrm{T}_{\mathrm{cs}}$ with cycles, shown in Fig. 4, presents a degradation of $\approx 0.25 \mathrm{~K}$,

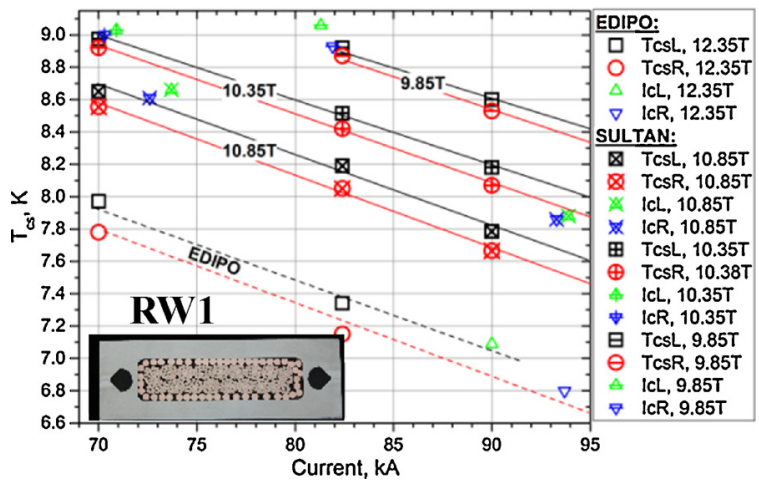

Fig. 3. $I_{c}$ and $T_{c s}$ results after the WUCD for the RW1 sample.

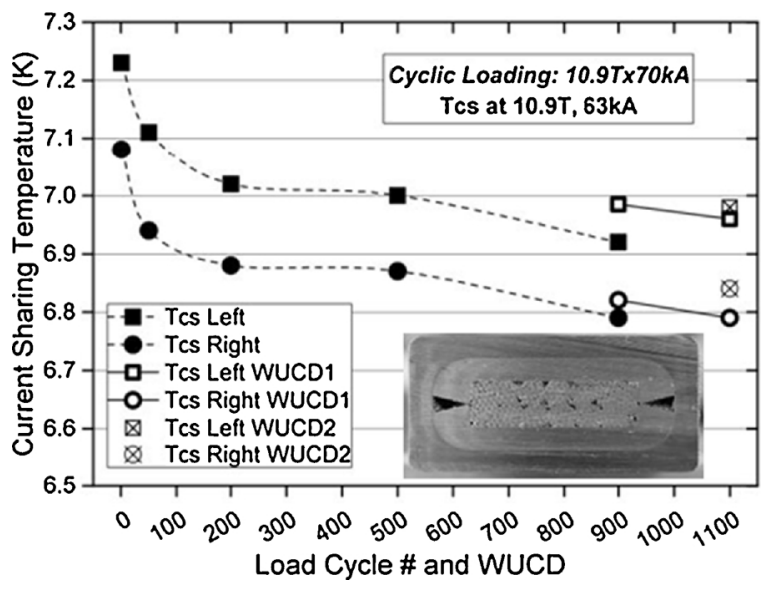

Fig. 4. Evolution of $\mathrm{T}_{\mathrm{cs}}$ with load cycles measured on RW2 sample.

presumably due to a shift in the strain distribution. In fact, the index of the superconducting transition remains stable at $n=18$, excluding filament breakage as an explanation for the performance loss. For the RW2 sample, the effective strain is $\approx-0.40 \%$. The AC losses measured on the RW2 sample are very low, giving a coupling loss constant $n \tau$ $\approx 50 \mathrm{~ms}$. This suggests that this layout could also be used for a CS conductor. 


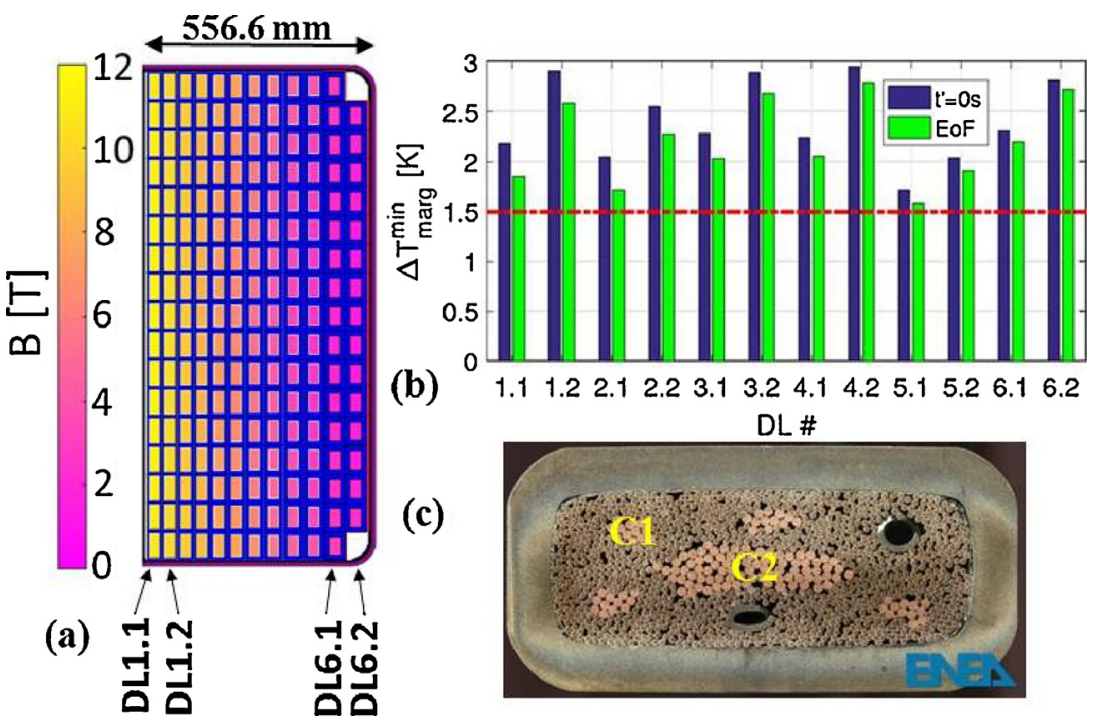

Fig. 5. (a) TF WP\#2 configuration (b) $\Delta T_{m \text { arg }}^{\min }$ in all layers before the plasma burn start (blue bars, on the left), and at EoF of a periodic pulse (green bars, on the right) (c) Cross-section of the SC conductor. (For interpretation of the references to colour in this figure legend, the reader is referred to the web version of this article.)

\section{2. $T F W P \# 2$ design}

WP\#2, sketched in Fig. 5(a), also has a layer-wound, graded structure, but based on six $\mathrm{Nb}_{3} \mathrm{Sn}$ double-layers (DL), and a WR CICC concept. The total number of turns is 202 , with a conductor operating current of $\mathrm{I}_{\mathrm{op}}=70.8 \mathrm{kA}$ at $4.5 \mathrm{~K}$ and $12.3 \mathrm{~T}$. The approach to the design of the cables is represented in Fig. 5(c) [16]: the segregated copper is either distributed within the cable bundle, with $1 \mathrm{~mm}$ diameter wires, or within the cores of the petals (C1), with $1.5 \mathrm{~mm}$ diameter wires, or within the overall cable core (C2), again with $1.5 \mathrm{~mm}$ diameter wires. The cable is formed with six petals around core C2, but two out of the six petals have a spiral (low-impedance cooling channels) as a core. The main geometrical parameters of TF WP\#2 are summarized in Table 2. The target void fraction in the cable bundle region is $0.26 \%$ for all conductors.

Comprehensive thermo-hydraulic analyses were carried out on the WP\#2 design assuming the NH load defined in [12]. The model, described in greater detail in [17], includes the 1D flow of supercritical He in each of the thermally coupled layers of the WP and in the casing cooling channels (CCC), and the heat conduction in the casing and the external cryogenic circuits for both WP and casing with quench lines. Moreover, the model accounts for both Joule heating from eddy currents in the casing, and AC losses in the cable induced by the fast discharge triggered by the quench protection system. Friction factor correlations and heat transfer values used in the model are validated on experimental tests [18]. The nominal DEMO pulsed scenario [19] is assumed for simulations. As shown in Figure 5(b), in normal operating conditions at the end of flat-top (EoF) the $\Delta T_{\mathrm{marg}}^{\mathrm{min}}$ requirement of $1.5 \mathrm{~K}$ is satisfied in all the conductors. The NH is responsible for a reduction of $\Delta T_{\operatorname{marg}}^{\mathrm{min}}$ of between 0.1 and $0.4 \mathrm{~K}$, depending on the distance from the plasma.

In off-normal operation, the design satisfies the maximum $\mathrm{T}_{\mathrm{hs}}$ criterion of $150 \mathrm{~K}$, even when considering a collapse of the relief channels in the WP and blockages of selected CCCs at critical locations.

Mechanical loads on WP\#2 have been analyzed using the Stress Recovery Tool (SRT) [20]. This method is designed to obtain reliable structural results from simplified models, with consistent time-saving with respect to full-detailed analyses. A hierarchical approach is envisioned: complete analysis of the TF coil D shape is conducted using a simplified model that adopts smeared properties for the mechanical analysis of the WP; results are then transferred to a full detailed model of a slice of WP so that an accurate representation of the stress state can be calculated.

In the casing, the stress hot spots are located under the OIS junctions $(800 \mathrm{MPa})$ and on the sharp edges of straight section of the inner leg (750 MPa), both higher than the allowable value (667 MPa). The local analysis shows that the average stress computed by the SRT is always below the allowable value (with a safety factor $\sim 1.6$ ), whereas the peak stress exceeds the limits in the inner corner of the jacket. The shear stress for insulation also exceeds the limits (54 MPa, that is $2 / 3$ of the ultimate strength of the insulating material) in a few critical locations, with a peak of $98 \mathrm{MPa}$.

To demonstrate the technological feasibility of large-size, high current conductors relevant to DEMO magnets, a short-length sample was manufactured and tested in the EDIPO facility [16]. The Cable-inConduit Conductor (CICC) was designed to satisfy the 2013 reference baseline [5], i.e. to operate at $81.7 \mathrm{kA}$ in an effective field of about $13 \mathrm{~T}$

Table 2

TF WP\#2 reference conductor design. Where different, the values for the first and second layer of the same. DL are separated by "/".

\begin{tabular}{|c|c|c|c|c|c|c|}
\hline Data & DL1 & DL2 & DL3 & DL4 & DL5 & DL6 \\
\hline N. SC strands $(D=1 \mathrm{~mm})$ & 720 & 360 & 270 & 180 & 120 & 120 \\
\hline Strand Cu:nonCu & 1 & 1 & 1 & 1 & 1 & 1 \\
\hline N. Cu strand $(D=1 \mathrm{~mm})$ & 360 & 720 & 540 & 630 & 690 & 690 \\
\hline N. Cu strand $(D=1.5 \mathrm{~mm})$ & 108 & 48 & 138 & 120 & 84 & 84 \\
\hline Conductor width $[\mathrm{mm}]$ & 68.9 & 68.9 & 68.9 & 68.9 & 68.9 & 68.9 \\
\hline Conductor height [mm] & 33.8 & 34.6 & 37.6 & 41.4 & 44.3 & 49.6 \\
\hline Jacket thickness [mm] & 3.9 & 5.3 & 6.8 & 8.4 & 10.0 & 11.7 \\
\hline Turn length $[\mathrm{m}]$ & $44 / 44.3$ & $44.5 / 44.8$ & $45 / 45.3$ & $45.5 / 45.8$ & $46.1 / 46.4$ & $46.7 / 47.1$ \\
\hline \# turns & $17 / 17$ & $17 / 17$ & $17 / 17$ & $17 / 17$ & $17 / 17$ & $17 / 15$ \\
\hline Hydraulic length [m] & $748 / 753$ & $757 / 761$ & $765 / 770$ & $774 / 779$ & $784 / 789$ & $794 / 706$ \\
\hline
\end{tabular}




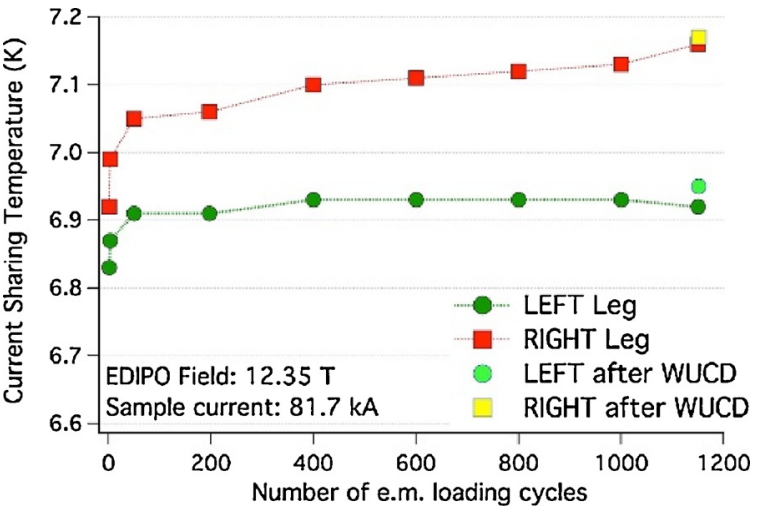

Fig. 6. $\mathrm{T}_{\mathrm{CS}}$ vs. number of electromagnetic loading cycles for the two legs of TF WP\#2 sample. After the last cycle, an additional measurement is done after WUCD.

(with peak field of $13.5 \mathrm{~T}$ ), and up to a temperature of at least $6.5 \mathrm{~K}$. The evolution of the $\mathrm{T}_{\mathrm{CS}}$ over the electromagnetic load cycles is shown in Fig. 6. It is worth noting that no degradation of $\mathrm{T}_{\mathrm{CS}}$ occurs, over more than 1000 cycles.

The behavior of $\mathrm{T}_{\mathrm{CS}}$ vs. the operating current at different background magnetic field values was compared with the $\mathrm{T}_{\mathrm{CS}}$ curves computed starting from $\mathrm{I}_{\mathrm{c}}(\varepsilon, \mathrm{T}, \mathrm{B})$ data collected at the University of Twente on the strands used [14]. The comparison provides an effective total strain value in the range: $-0.55 \% \div-0.50 \%$, that corresponds to the design target.

AC losses were measured by using both calorimetric and magnetization methods [16,21], with the magnetic field orientation either perpendicular or orthogonal to the wide conductor side. The energy deposited per cycle and per unit volume is rather high, with a pronounced difference depending on the orientation. Moreover, only a minor reduction in the losses is observed with cycling, thus differing from the behavior normally exhibited by ITER conductors [22]. These unclear aspects deserve wider investigation on further samples.

\subsection{TF WP\#3 design}

The TF WP\#3 consists of nine double pancakes (DP) wound in nine turns per pancake based on $\mathrm{Nb}_{3} \mathrm{Sn}$ W\&R fabrication process [23]. The TF conductor proposal is an ITER-like $88 \mathrm{kA}$ CICC, with a central spiral inserted in a thick square SS jacket to compensate the absence of radial plates. Fig. 7 shows the TF WP\#3 and the conductor, whose main characteristics are summarized in Table 3.

Thermal-hydraulic studies have been carried out both in normal and off-normal operating conditions [24,25].

The simulations of a two-hour burn, focused on both central and lateral clock-wise (CW) pancakes, allowed to assess the $\Delta T_{\mathrm{mar}}^{\mathrm{min}}$. In presence of CCC the $1.5 \mathrm{~K}$ criterion is respected, whereas it slightly fails in
Table 3

TF WP\#3 reference conductor design.

\begin{tabular}{|c|c|}
\hline Parameter & Value \\
\hline Operating current $[\mathrm{kA}]$ & 88 \\
\hline Peak operating field $[\mathrm{T}]$ & 12.27 \\
\hline Strand diameter $[\mathrm{mm}]$ & 0.984 \\
\hline Cr plating $[\mu \mathrm{m}]$ & 2 \\
\hline Number SC strands & 828 \\
\hline Number Cu strands & 790 \\
\hline Cable size $[\mathrm{mm}]$ & 43.62 \\
\hline Void fraction in cable [\%] & 30 \\
\hline Jacket thickness $[\mathrm{mm}]$ & 9.43 \\
\hline Hydraulic length $[\mathrm{m}]$ & 408 \\
\hline
\end{tabular}

the central CW if CCC are not included.

If the radial thermal coupling from turn to turn and between adjacent pancakes is implemented in the model, $\Delta T_{\operatorname{marg}}^{\min }$ decreases by $\sim 0.07 \mathrm{~K}$, showing that the effect of radial thermal exchanges inside the winding pack is limited.

Several quench scenarios were simulated, considering a heat deposition either on the internal turn (high field and low $\Delta T_{\operatorname{marg}}^{\min }$ ) for both central and lateral pancakes, or at the middle of hydraulic length (lower field and higher $\Delta T_{\mathrm{marg}}^{\mathrm{min}}$ ) for the central pancake. In all cases, the disturbance was one meter long and lasted $100 \mathrm{~ms}$, and the quench was initiated with twice the minimum quench energy. The $\mathrm{T}_{\mathrm{hs}}$ criterion was satisfied for all cases, except when the quench was initiated at middle of conductor, at rather low field.

A mechanical analysis similar to the one presented for WP\#1 has been done for WP\#3 [9]. On the inboard leg (point 2 of Fig. 8(a)) of $\mathrm{WP} \# 3$, the sum of the membrane stress plus bending (914 MPa) calculated over the critical path exceeds the static limit of $867 \mathrm{MPa}$ (see Fig. 8 (b)), whereas the membrane stress is within the limits. On the outboard leg (point 1 of Fig. 8(a)) all stresses are safely below the allowable values. In addition, a detailed mechanical analysis of the cumulative radial stress in the conductor jacket [23] was performed. The static stress criteria are fulfilled by increasing the jacket inner radius from $2 \mathrm{~mm}$ to $6 \mathrm{~mm}$, thus decreasing the bending of the conductor walls.

The short length sample of the WP\#3 conductor is under manufacture and will be tested in 2018 .

\section{Preliminary TF quench protection circuit}

Four alternative topologies (A, B, C and D) have been proposed for the DEMO TF quench protection circuit (QPC) [26], as shown in Fig. 9. The main differences are in the grounding system (ITER-like or JT-60SA like) and in the connection of the discharge resistors, that can be in parallel to the QPC circuit breaker or to the coil. In all cases, when the QPC intervenes, the terminal-to-ground voltage is half the voltage across the coil.

The numerical simulations performed provide the voltage

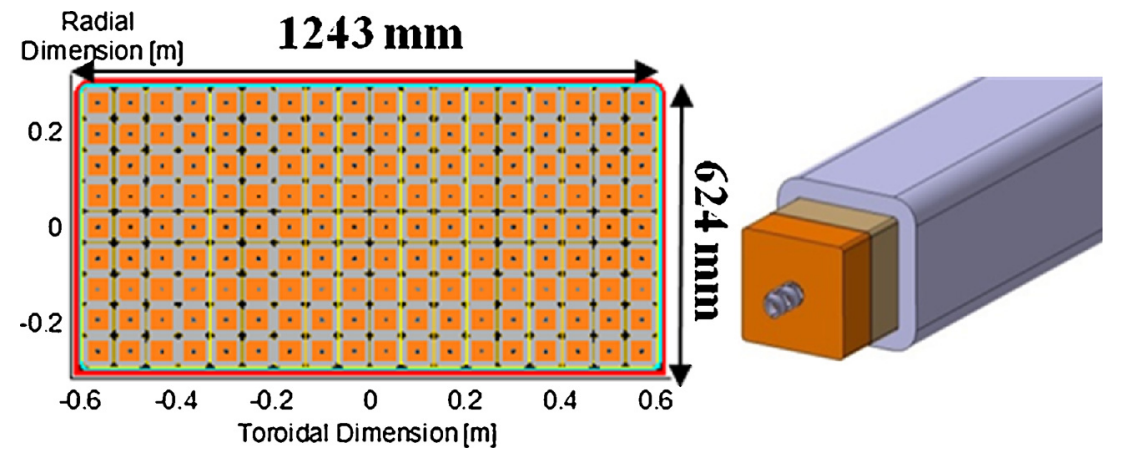

Fig. 7. TF WP\#3 reference structure and conductor design. 


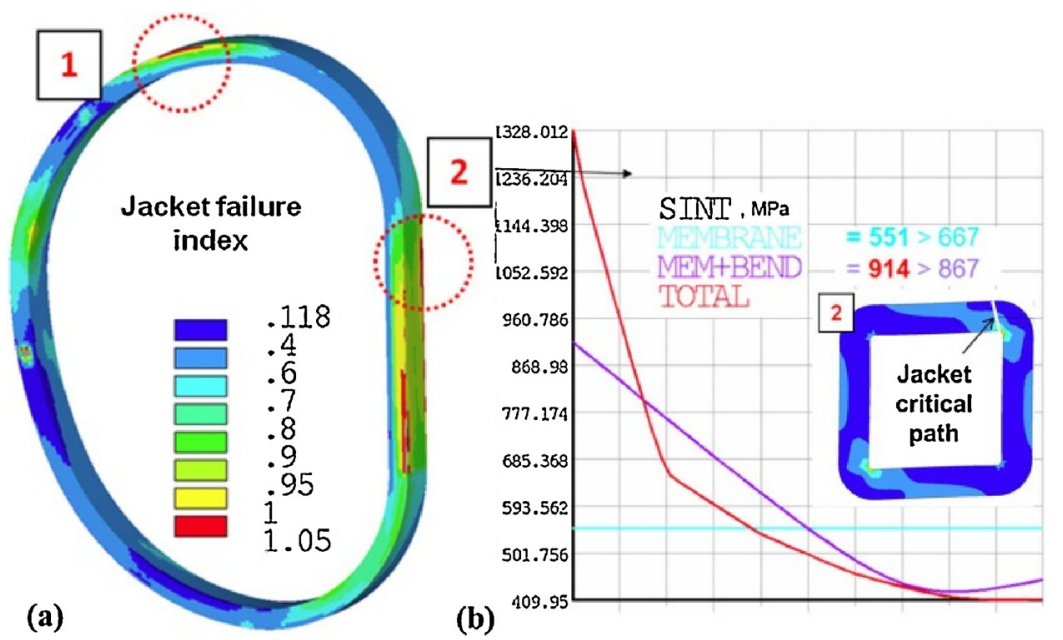

Fig. 8. (a) Structurally critical locations in TF WP\#3 (b) Jacket critical path with corresponding results.

waveforms at the coil terminals, across the coils and the Joule integral parameter, $\mathrm{i}^{2} \mathrm{t}$, in the coils during the discharge and in several fault conditions, for cases where the set of $18 \mathrm{TF}$ coils are lumped into 18 or 9 sectors.

The topologies show relative merits; with topologies C and D, the overall resistance remains the same in all the operating conditions, thus the discharge time constant is not modified and therefore there is not an increase in the $\mathrm{i}^{2} \mathrm{t}$. Moreover, in the majority of the operating conditions identified, the lowest terminal-to-ground voltage is obtained with topology D. Just to give an example, for 18 sectors, in a critical operating condition (QPC intervention failure and ground fault opposite to faulted QPC) the terminal-to-ground voltages for topologies A $(12.1 \mathrm{kV})$, B $(11.6 \mathrm{kV})$ and $\mathrm{C}(11.8 \mathrm{kV})$ are 2.5 times higher than in the case of regular QPC intervention $(4.4 \mathrm{kV})$, while it is two times this value with topology D $(8.8 \mathrm{kV})$. However, C and D topologies present an important limit; in fact, in case of untimely intervention of one QPC only, the
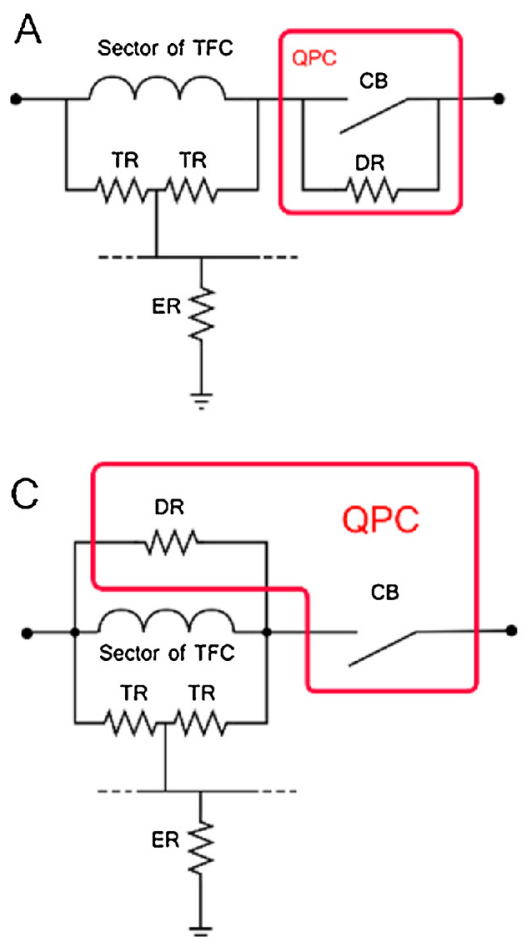

transient voltage peak applied to the coil, before the activation of all the others, could raise to very high values; thus the analysis is presently concentrated on A and B ones.

The 9 sectors option has been also studied due to the advantages in terms of cost and size of the protection system, penetrations in the tokamak building, bus-bars and current leads. The voltage peaks are doubled and exceed the limits presently assumed $(10 \mathrm{kV})$ for the TF coil insulation design; the adoption of resistors with variable resistance with temperature, as done both for ITER and JT-60SA, can be considered to evaluate the achievable voltage peak reduction.

\section{Central solenoid pre-conceptual design}

As for the TF, the CS should fulfill the geometrical and operational requirements defined by the systems code PROCESS [4]. The required geometry is a CS split into five modules, which should provide a total
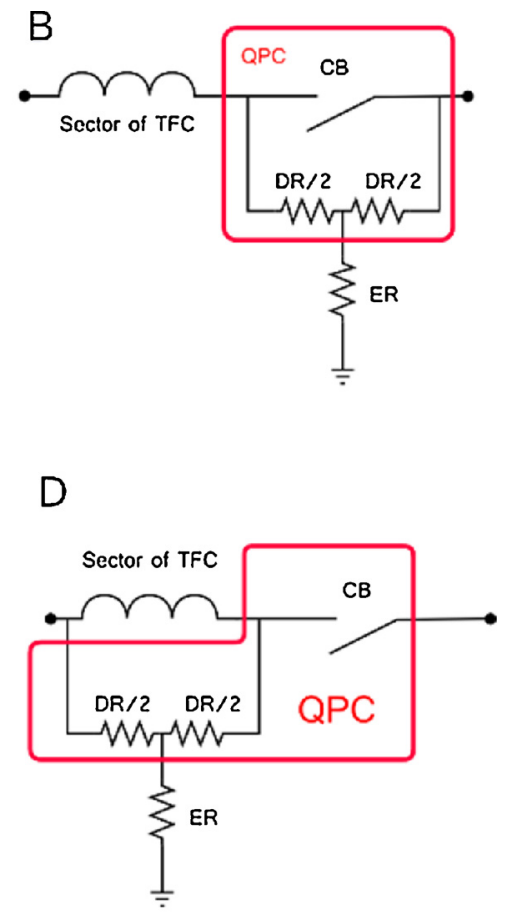

Fig. 9. Circuit topologies: A is ITER-like, B is JT-60SA like, C and D differ from A and B respectively for having the discharge resistor connected in parallel to the relevant TFC sector. 
Table 4

$\mathrm{Nb}_{3} \mathrm{Sn} \mathrm{CS}$ conductor and WP design.

\begin{tabular}{ll}
\hline Parameter & Value \\
\hline Conductor size [mm] & 61 \\
Conductor current [kA] & 55.5 \\
Jacket thickness [mm] & 12.8 \\
Number SC strands & 659 \\
Number Cu strands & 596 \\
Number of turns & 13 \\
Number of pancakes in the CS & 266 \\
External radius of the CS [m] & 3.2 \\
Internal radius of the CS [m] & 2.39 \\
Total strain in the coil [\%] & -0.515 \\
Maximum field on conductor [T] & 13.7 \\
Hoop stress [MPa] & 527.3 \\
Radial stress [MPa] & 31.3 \\
Vertical stress [MPa] & 135.9 \\
Tresca stress [MPa] & 663.2 \\
Central module inductance [H] & 6.02 \\
Dump time constant (for $\left.\mathrm{V}_{\max }=10 \mathrm{kV}\right)$ [s] & 16.7 \\
\hline
\end{tabular}

Table 5

Hybrid CS WP design.

\begin{tabular}{llllll}
\hline Sub-coil & Superconductor & $\mathrm{r}_{\mathrm{i}}(\mathrm{mm})$ & $\mathrm{d}_{\text {cond }}(\mathrm{mm})$ & $\mathrm{B}_{\text {peak }}(\mathrm{T})$ & $\mathrm{T}_{\text {cs }}(\mathrm{K})$ \\
\hline 1 & RE-123 & 1900.0 & 48.27 & 17.49 & 10.51 \\
2 & RE-123 & 2045.5 & 45.88 & 15.75 & 10.64 \\
3 & Nb3Sn & 2169.3 & 51.31 & 14.02 & 6.25 \\
4 & Nb3Sn & 2279.9 & 41.90 & 12.22 & 6.26 \\
5 & Nb3Sn & 2371.7 & 37.40 & 10.44 & 6.25 \\
6 & Nb3Sn & 2454.5 & 34.17 & 8.68 & 6.25 \\
7 & Nb3Sn & 2530.9 & 30.97 & 6.93 & 6.24 \\
8 & NbTi & 2600.8 & 38.95 & 5.43 & 6.25 \\
9 & NbTi & 2687.7 & 27.71 & 3.93 & 6.25 \\
10 & NbTi & 2750.1 & 27.46 & 2.64 & 6.25 \\
\hline
\end{tabular}

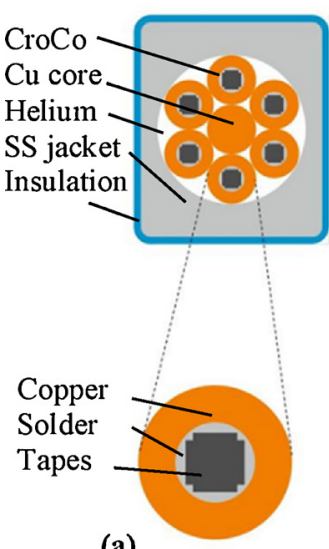

(a)

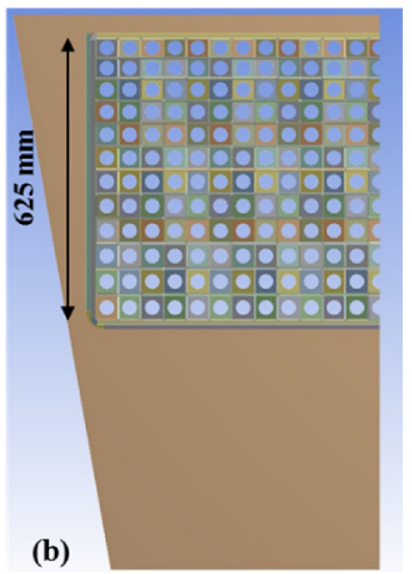

(b)
Fig. 10. (a) HTS Conductor based on CroCo strands, (b) TF WP based on HTS conductors.

magnetic flux of $\Phi_{\mathrm{CS}}=320 \mathrm{~Wb}$. Two designs are presented for the CS magnet, as reported below.

\section{1. $\mathrm{Nb}_{3} \mathrm{Sn}$-based CS design}

A first design based on a pancake wound $\mathrm{Nb}_{3} \mathrm{Sn}$ conductor has been proposed for the CS coils [23]. The external radius has been fixed to $3.2 \mathrm{~m}$ in order to take into account the gap between TF and CS, used to accommodate mechanical deformation of $\mathrm{TF}$ as well as pre-compression tie-plates. The design is based on the CSJA6 $\mathrm{Nb}_{3} \mathrm{Sn}$ strand, in order to exploit the experimental results obtained for ITER R\&D [27]. The main parameters of the CS WP and conductor are presented in Table 4.
Table 6

TF HTS-based reference conductor design.

\begin{tabular}{ll}
\hline Parameter & Value \\
\hline Number of CroCos & 6 around 1 copper core \\
Diameter of CroCo and Cu core [mm] & 10.4 \\
Conductor width [mm] & 38.2 \\
Conductor height [mm] & 45.1 \\
Total Copper in CroCo $\left[\mathrm{mm}^{2}\right]$ & 493.23 \\
Solder [mm $\left.{ }^{2}\right]$ & 56.41 \\
REBCO in REBCO tapes $\left[\mathrm{mm}^{2}\right]$ & 1.21 \\
Hastelloy in REBCO tapes $\left[\mathrm{mm}^{2}\right]$ & 45.00 \\
Space for Helium cooling[mm $\left.\mathrm{mm}^{2}\right]$ & 165.4 \\
Jacket (innermost layer) $\left[\mathrm{mm}^{2}\right]$ & 954.85 \\
\hline
\end{tabular}

The burn scenario has been simulated [24] considering $80 \mathrm{~s}$ premagnetisation, a two-hour burn, and a $10 \mathrm{~min}$ dwell period. Coupling and hysteresis AC losses have been computed in order to assess the temperature margin. The minimum value, found at the end of first turn and at the end of the dwell, was $1.42 \mathrm{~K}$ for $\mathrm{n} \tau=638 \mathrm{~ms}$, that is the time constant value deduced from ITER CS insert tests performed at CSMC Naka in 2015 [27]. The sensitivity analysis has shown that in order to meet the $1.5 \mathrm{~K}$ criterion, the $\mathrm{n} \tau$ would have to decrease to $250 \mathrm{~ms}$.

The AC losses during the breakdown CS current variation were also computed [28] considering a variation of the maximum external magnetic field of $1.7 \mathrm{~T} / \mathrm{s}$ over $0.8 \mathrm{~s}$ [29]. The $\Delta T_{\mathrm{marg}}^{\mathrm{min}}$ drops significantly below $1.5 \mathrm{~K}$ unless the $\mathrm{n} \tau$ is reduced to below $200 \mathrm{~ms}$. Considering the screening current effect in cable, which attenuates the external field variation inside the cable, the power deposited is highly reduced and the $\Delta T_{\operatorname{marg}}^{\min }$ is around the limit. The breakdown phase is a critical step from the point of view of the CS temperature margin and deserves further analysis.

\subsection{Hybrid CS design}

A second design has been proposed for the CS1 module, based on 10 layer-wound sub-coils using HTS, R\&W $\mathrm{Nb}_{3} \mathrm{Sn}$, and NbTi conductors in the high, medium and low field sections, respectively [30]. Analogous to the TF WP\#1 approach, a grading of SC and SS materials is considered. The hoop stresses and the vertical loads are included for determining the required SS cross-section in the WP. In respect to the $\mathrm{Nb}_{3} \mathrm{Sn}$-based solution, the use of HTS in the inner sub-coils allows to maintain the same magnetic flux of $320 \mathrm{Vs}$ in the central plane, with a higher magnetic field and a reduced outer radius of $2.813 \mathrm{~m}$.

For each sub-coil, made up by a double layer, the inner radius, $r_{i}$, the radial thickness of the conductor without insulation, $d_{\text {cond }}$, the peak magnetic field, $\mathrm{B}_{\text {peak }}$, and $\mathrm{T}_{\mathrm{cs}}$, are listed in Table 5 . The conductor operation current is $50.95 \mathrm{kA}$.

The required SS cross-section in the winding pack was determined through mechanical analyses, in which both the hoop stresses and the vertical loads were included. A graded design of the SS conduits is proposed since the hoop stress decreases significantly for increasing radii. The pre-magnetization current configuration is used to determine the required SS fraction for each double layer. The membrane stress across the thickness of the conduits ranges between 590 and $665 \mathrm{MPa}$ during this phase.

In order to estimate the required cross-section of the pre-compression tie plates, a global model including the five CS modules, the 100mm-thick buffer plates between the modules, and the pre-compression structure has been proposed. Assuming that the superalloy Nitronic 50 is used, the minimum radial thickness for the inner and outer tie plates is 139 and $87 \mathrm{~mm}$, respectively (assuming that the tie plates cover half of the actual perimeter of the CS winding pack).

Thermal-hydraulic analyses have been carried out on the HTS subcoils of the CS1 module up to the breakdown (BD), foreseen at $t=0.8 \mathrm{~s}$ after the start of the pulse [29]. The steep current change is expected to induce large AC losses in the SC, nevertheless $\Delta T_{\operatorname{marg}}^{\mathrm{min}}$ always stays above 
$3 \mathrm{~K}$.

\section{HTS activity for DEMO magnets}

Conductors based on High Temperature Superconductors (HTS) are under investigation to identify the benefits of this technology.

REBCO tapes have been characterized both in the virgin state and after the irradiation of fast neutrons with a fluence of up to $4 \cdot 10^{22}$ neutrons $/ \mathrm{m}^{2}$ [31]. The critical current $\mathrm{I}_{\mathrm{c}}$ initially increases but degrades at high fluence. First ideas on the degradation mechanisms have been developed but further studies (properties and microstructure) are needed for establishing the correlation between microstructure (pinning landscape, grain structure etc.) and radiation response.

A $60 \mathrm{kA}$ REBCO prototype conductor (based on a flat cable design) was tested at $5 \mathrm{~K}, 12 \mathrm{~T}$ in 2015 [32]. A $15 \%$ reduction of the $\mathrm{I}_{\mathrm{c}}$ was observed after the electro-magnetic cycling, mostly due to degradation at the cable edges.

A conceptual design of a TF WP based on a HTS flat cable was proposed in 2016 [33], but the $\mathrm{T}_{\mathrm{hs}}$ exceeded the $150 \mathrm{~K}$ criterion, and the structural analysis for the jacket reported safety factors for the maximum Tresca stress lesser than one. To overcome these issues, a new design has been proposed based on a round 6 Cross Conductor (CroCo) monolithic strands [34] (10.4 mm in diameter) around 1 copper core embedded in SS jacket, as shown in Fig. 10(a). The main design parameters are reported in Table 6 . The conductor is dimensioned to carry $47.6 \mathrm{kA}$ in a maximum field of $11.9 \mathrm{~T}$. The WP consists of 12 layers with 25 turns/layer, as sketched in Fig. 10(b). The cable dimension is unchanged for different layers, whereas the jacket thickness is constant in the toroidal direction and increases in the radial direction away from the plasma to deal with accumulated stresses. The results of thermal-hydraulic analyses are rather encouraging with $\mathrm{a} \Delta T_{\operatorname{marg}}^{\min }>10 \mathrm{~K}$ and $\mathrm{T}_{\mathrm{hs}}=154 \mathrm{~K}$ for the jacket, only slightly higher than the criterion. The 2D structural analysis for this design leads to stresses below the limits. The combined shear and compression stress in the turn insulation is also acceptable. Experimental tests on a smaller HTS CroCo sample [35] have demonstrated a performance of about 5 $\mathrm{kA}$ at $4.2 \mathrm{~K}, 12 \mathrm{~T}$.

Two new samples, based on a similar concept, but properly designed for the CS inner layers, are now under manufacture and will be tested in 2018.

\section{Conclusions}

The design of the magnet system for the DEMO tokamak is proceeding by exploring different solutions in terms of winding approaches (pancakes vs. layers) and manufacturing techniques (R\&W vs. W\&R $\mathrm{Nb}_{3} \mathrm{Sn}$ ), with a present focus on the TF and CS coils. Experimental results show that the degradation of the conductor performances with electromagnetic cycles is absent or limited compared to most of the large-size $\mathrm{Nb}_{3} \mathrm{Sn}$ conductors reported in literature. The effective strain is also consistently lower, with a consequent cost saving, due to the reduced amount of superconducting material required. Critical aspects in the mechanical structure and especially on the insulation have been identified, requiring further investigation and R\&D activity. Innovative solutions based on HTS tapes have also been proposed, in order to demonstrate their potential use in the CS coil and identify the benefits of these materials for future fusion power plants beyond DEMO.

\section{Acknowledgments}

This work has been carried out within the framework of the EUROfusion Consortium and has received funding from the EURATOM research and training programme 2014-2018 under grant agreement No 633053. The views and opinions expressed herein do not necessarily reflect those of the European Commission.

\section{References}

[1] F. Romanelli, Fusion Electricity, A roadmap to the realization of fusion energy, European Fusion Development Agreement, EFDA - Nov. 2012 - ISBN 978-3-00040720-.

[2] G. Federici, et al., DEMO design activity in Europe: progress and updates, Fus. Eng. Des. (2018), http://dx.doi.org/10.1016/j.fusengdes.2018.04.001 in press.

[3] L. Zani, et al., Overview of progress on the EU DEMO reactor magnet system design, IEEE Trans. Appl. Supercond. 26 (2016) 4204505.

[4] R. Wenninger, Reference design - 2015 April (EU DEMO1 2015) PROCESS full output, https://idm.euro-fusion.org/?uid = 2MDKFH.

[5] R. Wenninger, DEMO1 - November 2013 - PROCESS output, https://idm.eurofusion.org/?uid = 2MC995.

[6] K. Sedlak, et al., Design and R\&D for the DEMO toroidal field coils based on $\mathrm{Nb}_{3} \mathrm{Sn}$ react and wind method, IEEE Trans. Appl. Supercond. 2627 (2017) 4800105.

[7] Bruzzone, et al., A prototype conductor by React\&Wind method for the EUROfusion DEMO TF coils, IEEE Trans. Appl. Supercond. 28 (2018) 4202705.

[8] A. Panin, et al., Structural analysis of fusion magnets: engineering zooming on the superconductor strength, Fus. Eng. Des. (2017) submitted to.

[9] A. Panin, et al., Mechanical pre-dimensioning and pre-optimization of the tokamaks' toroidal coils featuring the winding pack layout, Fus. Eng. Des. 124 (2017) 77-81.

[10] K. Sedlak, et al., Thermal-hydraulic and quench analysis of the DEMO toroidal field winding pack WP1, Fus. Eng. Des. 124 (2017) 110.

[11] M. Lewandowska, et al., Thermal-hydraulic analysis of different design concepts of the LTS TF coil winding pack for EU-DEMO, Proceedings of the 2017 Int. Conf. on ELMECO \& AoS, 3-6.12.2017, Naleczow, Poland, 2018, http://dx.doi.org/10.1109/ ELMECO.2017.826776.

[12] L. Zani and U. Fischer, Advanced definition of neutronic heat load density map on DEMO TF coils (2014) https://idm.euro-fusion.org/?uid=2MFVCAv1.0.

[13] R. Zanino et al., Common approach for thermal-hydraulic calculations (2016) available at http://www.euro-fusionscipub.org/archives/eurofusion/commonoperating-values-for-demo-magnets-design-for-2016.

[14] V. Corato, et al., Common Operating Values for DEMO Magnets Designfor 2016, (2016) Available at: http://www.euro-fusionscipub.org/archives/eurofusion/ common-operating-values-for-demo-magnets-design-for-2016-2 ..

[15] P. Bruzzone, et al., Upgrade of operating range for SULTAN test facility, IEEE Trans. on Appl. Supercond. 12 (2002) 520-523.

[16] Muzzi, et al., Design, manufacture, and test of an 80 kA-Class $\mathrm{Nb}_{3} \mathrm{Sn}$ cable-InConduit conductor with rectangular geometry and distributed pressure relief channels, IEEE Trans. Appl. Supercond. 27 (2017) 4800206.

[17] L. Savoldi, et al., Performance analysis of a graded winding pack design for the EU DEMO TF coil in normal and off-normal conditions, Fus. Eng. Des. 124 (2017) 45-48.

[18] R. Bonifetto, et al., Thermal-hydraulic test and analysis of the ENEA TF conductor sample for the EU DEMO fusion reactor, IEEE Trans. Appl. Supercond. 28 (2018) 4205909.

[19] B. Meszaros, EU DEMO1 2015 Plasma and equilibrium description, https://idm. euro-fusion.org ? ?uid = 2LJFN7

[20] M. Biancolini, et al., Mechanical analysis of the ENEA TF coil proposal for the EU DEMO fusion reactor, IEEE Trans. Appl. Supercond 28 (2018) 4901405.

[21] K. Yagotintsev, A. Nijhuis, AC loss, interstrand resistance and mechanical properties of prototype EU DEMO TF conductors up to 30000 load cycles, Supercond. Sci. Technol. 31 (2018) 025010.

[22] Breschi,, et al., Results of the TF conductor performance qualification samples for the ITER project, Supercond. Sci. Technol. 25 (2012) 095004.

[23] A. Torre, et al., EU-DEMO TF and CS magnet systems design and analyses performed at CEA, IEEE Trans. Appl. Supercond. 27 (2017) 4900705.

[24] R. Vallcorba, et al., Thermohydraulic analysis on CEA concept of TF and CS coils for EU-DEMO, IEEE Trans. Appl. Supercond. 28 (2018) 4202605.

[25] Q. Le, Coz et al. Quench simulation of a DEMO TF coil using a quasi-3D coupling tool, IEEE Trans. Appl. Supercond. 28 (2018) 4203105.

[26] Maistrello, et al., Studies on the TF circuit of DEMO, https://idm.euro-fusion.org/? uid $=2$ M8RJB\&version $=$ v3.0.

[27] N. Martovetsky, et al., ITER central solenoid insert test results, IEEE Trans. Appl. Supercond. 26 (2016) 4200605.

[28] L. Zani, et al., Status of CEA magnets design tools and applications to EU DEMO PF and CS magnets, IEEE Trans. Appl. Supercond. 28 (2018) 4902205.

[29] M. Mattei, DEMO AR $=3.1$ preliminary Breakdown magnetic analyses, 11/08/ 2015.

[30] R. Wesche, et al., DEMO central solenoid design based on the use of HTS sections at highest magnetic field, IEEE Trans. Appl. Supercond. 28 (2018) 4203605.

[31] D.X. Fischer, et al., The effect of fast neutron irradiation on the superconducting properties of REBCO coated conductors with and without artificial pinning centers, Supercond. Sci. Technol. 31 (2018) 044006.

[32] D. Uglietti, et al., Test of $60 \mathrm{kA}$ coated conductor cable prototypes for fusion magnets, Supercond. Sci. Technol. 28 (2015) 124005.

[33] R. Heller, et al., Conceptual design improvement of a toroidal field coil for EU DEMO using high-Temperature superconductors, IEEE Trans. Appl. Supercond 26 (2016) 4201105

[34] M.J. Wolf, et al., HTS CroCo: a stacked HTS conductor optimized for high currents and long-Length production, IEEE Trans. Appl. Supercond. 26 (2016) 6400106.

[35] M.J. Wolf, et al., High current densities in small HTS CrossConductors at low temperatures and high magnetic fields, IEEE Trans. Appl. Supercond. (2017). 